\title{
Die Bedeutung von Musiksammlungen für die Entstehung der Musikwissenschaft
}

Obwohl die Dresdner Musikabteilung mit ihrer nunmehr 200-jährigen Geschichte zu den ältesten eigenständigen Musiksammlungen innerhalb von Bibliotheken gehört, ist sie viel jünger als die zunächst kurfürstliche, dann königliche Bibliothek selbst. ${ }^{1}$ Sie geht auf die Zusammenlegung der musikbezogenen Bücher und ihre Verzeichnung in einem gemeinsamen Katalog zurück, die der Bibliothekar Friedrich Adolf Ebert im Rahmen einer allgemeinen Bestandsrevision 1815/16 unternahm. ${ }^{2}$ Damit stellte sich die Dresdner Musiksammlung zunächst als eine klassische Musikbuchsammlung dar, wie sie als Teilbereich einer Artes liberales-Abteilung auch schon in einer mittelalterlichen Klosterbibliothek existierte.

Größere Musikalienbestände gelangten erst ab der Mitte des 19. Jahrhunderts durch Nachlässe, Zensur-Exemplare, Ankäufe und Deposita - also im Wesentlichen unsystematisch - in die Sammlung. Die bedeutenden Zeugnisse der sächsischen Musikgeschichte aber, für die die Abteilung heute so berühmt ist, kamen überhaupt erst ab den 1890er Jahren hinzu: Auf die umfangreiche königliche Privat-Musikaliensammlung (inklusive des berühmten Schranks II) folgten ab 1908 die nicht mehr genutzten Noten der Hofkirche, ab 1930 die Bestände der Staatsoper. ${ }^{3}$

Eine Geschichte wie diese erinnert daran, dass die uns heute so vertraute und unverzichtbare Existenz von Musiksammlungen, das Aufbewahren und Erschließen von Musikalien - gemeinsam mit musikbezogenen Büchern - in Bibliotheken zum Zwecke der Sammlung, Dokumentation und Erforschung alles andere als selbstverständlich ist. Der ,natürliche ' Lebensraum für Musikmanuskripte und -drucke sind schließlich die Institutionen und Orte, in denen aus ihnen musiziert wird: Kapellen, Orchester, Opernhäuser, private Kammern und Haushalte. Was nicht mehr gebraucht und geschätzt wird (ein Stadium, in das über Jahrhunderte große Teile des Repertoires

11556 Gründung durch Kurfürst August I. (1574 erster Katalog), 1788 als öffentliche kurfürstliche Bibliothek, 1806 Königliche Öffentliche Bibliothek, 1918 Sächsische Landesbibliothek, seit 1996 Sächsische Landesbibliothek - Staats- und Universitätsbibliothek (SLUB) Dresden.

2 Die Arbeitsschritte und Vorgehensweise sind dokumentiert in: Friedrich Adolf Ebert, Diarium über meine Arbeiten [...] auf der Königl. Bibl. zu Dresden 1814-1822, D-Dl: Ms. autogr. I A 3d, hier zitiert nach Typoskript, S. 61-64, 67, 69. Der Katalog Musica ist in D-Dl unter der Signatur II Ea 444 erhalten.

3 Zur Geschichte der Musikabteilung der SLUB siehe Ortrun Landmann und Wolfgang Reich, Führer durch die Musikabteilung der Sächsischen Landesbibliothek zu Dresden, Dresden 1980. 
relativ rasch eintraten), ${ }^{4}$ verstaubt in der Ecke oder wird - namentlich bei Platzmangel - umgenutzt (etwa für Bucheinbände) oder weggeworfen.

Die Entscheidung für das Aufbewahren, die Archivierung und gar bibliothekarische Erschließung von Musikalien, die keinem praktischen Interesse mehr dienen, setzt indes bereits ein historisches Bewusstsein voraus - ein lokal- bzw. institutionshistorisches oder sogar schon ein musikhistorisches: für die Geschichte der eigenen Institutionen, die Bedeutung eines Bestandes oder eines Komponisten. Mit der Übertragung ausgemusterter Musikalien an ein Archiv oder eine Bibliothek ändert sich ihr Status grundlegend: Sie verlieren ihre ursprüngliche Funktion als Musiziervorlage und werden zu einem historischen Dokument bzw. gewinnen eine potenzielle Bedeutung für Forscher und Gelehrte. ${ }^{5}$ Daraus ergibt sich überdies eine Änderung des Benutzerprofils. Benutzungsweisen, Sortierungs- und Aufbewahrungsstrategien wandeln sich.

Die Entstehung und Pflege von Musiksammlungen in Bibliotheken (oder Archiven) - also der Ausbau von reinen Musikbuch- zu Buch- und Musikaliensammlungen - muss folglich in einem denkbar engen reziproken Zusammenhang mit der Entwicklung des Faches Musikwissenschaft gesehen und beschrieben werden. Denn erst musikhistorisches Denken sowie ein im engeren Sinne musikwissenschaftliches (und nicht länger nur musiktheoretisch oder kompositionspädagogisch motiviertes) Interesse an Kompositionen erzeugte auch ein Interesse an der Aufbewahrung aus dem Repertoire gefallener Musik. Umgekehrt ermöglichen erst nennenswerte Musiksammlungen das Schreiben von Musikgeschichte(n). An der Wiege der historischen Musikwissenschaft stand folglich die aus den praktischen Zusammenhängen gelöste Musikaliensammlung.

Ist diese These als solche auch kaum überraschend, hat sie doch noch kaum eingehendere Betrachtung erfahren. ${ }^{6}$ Und so wird es im Folgenden vor allem darum gehen, ihr durch Beispiele und eine gewisse Systematisierung Gestalt und Perspektive zu geben.

Der zeitliche Rahmen der Darstellung umfasst im Wesentlichen die Inkubations- und Etablierungszeit der Musikwissenschaft als universitäres Fach bis ins erste Drittel des 20. Jahrhunderts hinein. Die Dresdner Sammlung samt ihren Protagonisten und Benutzern wird dabei als Referenzbeispiel dienen und durch eingeschaltete Vergleiche mit anderen Institutionen und Personen kontextualisiert werden. Für den folgenden Text gilt eine Beschränkung auf wissenschaftliche

$4 \mathrm{Zu}$ diesen Zusammenhängen Melanie Wald-Fuhrmann und Klaus Pietschmann, Der Kanon der Musik. Theorie und Geschichte. Ein Handbuch, München 2013. Mit Quellenverlusten hat sich besonders Martin Staehelin beschäftigt, siehe etwa „Gestalt und Entstehung musikalischer Quellen im 15. und 16. Jahrhundert“, in: Quellenstudien zur Musik der Renaissance 3, hrsg. von Martin Staehelin, Wiesbaden 1998, sowie „Die Erforschung mittelalterlicher Musikfragmente. Ein Beitrag zur Revision unserer musikgeschichtlichen Vorstellungen“, in: Bibliothek und Wissenschaft 30 (1997), S. 26-40.

5 Ein besonders drastisches Beispiel dafür ist die abrupte Beendigung aktiver Kasseler höfischer Musikpflege durch Wilhelm IX./I. (Regierungszeiten 1785-1806 und 1813-1821): Dieser löste sämtliche höfische musikalische Institutionen auf und überstellte sämtliche Musikalien an die Bibliothek. Dazu Brigitte Pfeil, „Von landgräflicher Hofmusik bis zu moderner, Musica practica‘. Musikhandschriften und Musikdrucke im Bestand der Universitätsbibliothek Kassel - Landesbibliothek und Murhardsche Bibliothek der Stadt Kassel“, in: Musiksammlungen in den Regionalbibliotheken Deutschlands, Österreichs und der Schweiz, hrsg. von Ludger Syré, Frankfurt a. M. 2015, S. 211-224, bes. S. 218.

6 Den Zusammenhang zwischen der Entstehung eigenständiger Musiksammlungen an Bibliotheken und dem Aufkommen der Musikwissenschaft „als quellenbasierte historisch-kritische Wissenschaft“ konstatierte jüngst auch Sven Limbeck, „Schichtung und Profil. Die Musiksammlung der Herzog August Bibliothek Wolfenbüttel und ihre Provenienzen“, in: Syré, Musiksammlungen 2015 (wie Anm. 5), S. 375-389, hier S. 377. 
National-, Landes- und Universitätsbibliotheken und eine Fokussierung auf die Musikalien-, nicht die Bücherbestände, da hier der eigentliche historische Erklärungsbedarf und Erkenntniswert für die zugrunde liegende Fragestellung besteht. Die Schneisen, die dabei zu legen sind, entsprechen den auch heute noch für jede Musiksammlung zentralen Aufgaben des Sammelns und Aufbewahrens - Erschließens - Erforschens - Vervielfältigens. Dabei geraten immer wieder auch Wechselwirkungen zwischen den Sammlungen und dem sich konturierenden Fach in den Blick.

\section{Sammeln und Aufbewahren}

Die Gründungsgeschichten und impulsgebenden Motivationen für die Entstehung von eigenständigen Musikabteilungen sind einigermaßen divers. ${ }^{7} \mathrm{Zu}$ ihnen gehört aber fast immer die Übertragung eines einst praktisch genutzten Musikalienbestands von der entsprechenden Musikinstitution in die Bibliothek mit dem Zwecke der dauerhaften Aufbewahrung und historischen Dokumentation. Dass die Wahl für einen Sammlungskontext mehrheitlich und zunehmend auf die Bibliothek fiel und weniger auf das Archiv oder einen ganz eigenen Aufbewahrungskontext (wie ihn Moritz Fürstenau lange Jahre für Dresden favorisiert hätte), stellt bereits eine erklärungsbedürftige historische Entwicklung dar. Die wahrgenommene Verwandtschaft mit dem Buch dürfte sich wohl aus dem der Musik neu zugesprochenen (Kunst-)Werk- und Textcharakter herleiten lassen. ${ }^{8}$

Die historische Bedeutung eines Bestandes versteht sich indes nicht von selbst, sie muss zuvor erst diskursiv geschaffen werden - etwa durch Denkschriften oder Zeitschriftenartikel. Der generelle Historismus gerade der zweiten Hälfte des 19. Jahrhunderts bot dafür in vielen Fällen eine geeignete Grundlage, auf der auch ein musikhistorisches Interesse aufsetzen konnte. ${ }^{9}$

Die ,Umlagerungen' von Musikbeständen aus einem praktischen in einen bibliothekarischen Kontext konnte auf verschiedene Weise geschehen: Für Dresden - wie für die meisten Hofbibliotheken - gilt, dass die zur Hofmusik gehörenden, aber auf die verschiedenen musikalischen Institutionen und Privatpersonen verteilten Bestände zusammengeführt und dabei zugleich dem praktischen Kontext entzogen wurden. Sie verblieben aber im Bereich des Hofes. Eigentumsverhältnisse waren von der Umlagerung nicht betroffen.

Hier geht es also zunächst nur um die Aufbewahrung und den Erhalt schon vorhandener Bestände; Hell nannte Vorgänge dieser Art „passive Erwerbungen“. ${ }^{10}$ Die ersten Musikbibliothe-

7 Neben den jeweiligen Passagen in den Orts-Artikeln der MGG sowie punktuell existierenden Abrissen der eigenen Geschichte in der Verantwortung der Sammlungen bzw. Bibliotheken selbst sei auf die zahlreichen, wenngleich keinem gemeinsamen Raster verpflichteten Fallbeispiele bei Syré, Musiksammlungen 2015 (wie Anm. 5) hingewiesen.

8 Hier schließe ich mich der sehr plausiblen Deutung von Thomas Leibnitz in seinem Beitrag für diesen Band an („Musikbibliothek im europäischen Kontext. Zur Entstehung der Musiksammlung der Österreichischen Nationalbibliothek").

9 Siehe grundsätzlich zum Entstehen eines musikhistorischen Bewusstseins und zu dessen Inhalten und Strukturen in Deutschland: Frank Hentschel, Bürgerliche Ideologie und Musik. Politik der Musikgeschichtsschreibung in Deutschland 1776-1871, Frankfurt a. M. 2006.

10 Helmut Hell, „Die Musikabteilung. Stationen ihrer Geschichte“, in: Schätze wieder vereint. Die Zusammenführung der historischen Sonderabteilungen der Staatsbibliothek zu Berlin, Wiesbaden 1999, S. 8-19, hier S. 10. 
kare waren dann auch verschiedentlich einfach die Kustoden der Kapellsammlungen, etwa Otto Kade in Schwerin, die ihre Aufgabe oft unentgeltlich neben ihrer eigentlichen Bestallung als Hofmusiker ausübten.

Dem kann sich eine dezidierte Sammlungsstrategie anschließen mit dem Ziel, den Kernbestand systematisch zu ergänzen und zu erweitern. (Im engeren Sinne kann man erst jetzt von einer - bewusst geplanten und gepflegten - Sammlung im Unterschied zu einer zufällig und beiläufig zusammengekommenen Menge von Dokumenten sprechen.) Ab einer bestimmten Größe entwickeln Sammlungen allerdings regelmäßig eine Anziehungskraft auf kleinere verwandte Bestände.

Während die Dresdner Sammlung sich in erster Linie einer Aufbewahrungs-Motivation verdankt, ${ }^{11}$ kann eine Musiksammlung wie diejenige der Berliner Staatsbibliothek als Beispiel für eine Sammlungs-Motivation gelten: Auch hier lagern zwar mittlerweile zentrale Musikalienbestände der preußischen Kurfürsten und Könige. Vorausgegangen waren aber Ankäufe privater Musiksammlungen: 1824/25 derjenigen des Hallenser Universitätsmusikdirektors Johann Friedrich Naue und 1841 dann derjenigen von Georg Poelchau, die den Anlass für die organisatorische Abtrennung einer Musiksammlung und die Einstellung eines ersten eigenen Musik-Kustoden gab, der die Sammlung dann systematisch im durch Poelchau vorgegebenen Profil ausbaute. ${ }^{12}$

Private Musik-Sammler wie Poelchau gab es vor allem in der ersten Hälfte des 19. Jahrhunderts einige (s. Tabelle 1): ${ }^{13}$ Unabhängig von institutionellen Rahmenbedingungen konnten sie allein aufgrund persönlicher Interessen und Einschätzungen festlegen, dass und welche Quellen aufbewahrenswert waren. Damit können sie als frühe Verstärker eines im Entstehen begriffenen musikhistorischen Bewusstseins gelten und arbeiteten zugleich der späteren Institutionalisierung von Musiksammlungen zu. So scheint es nur folgerichtig, dass Privatsammlungen dann ab der zweiten Hälfte des 19. Jahrhunderts in staatliche Sammlungen eingingen und insgesamt ein Zentralisierungsprozess zu beobachten ist.

Die Profile privater und institutionell gewachsener Sammlungen lassen sich klar voneinander

11 Die zunächst erfolgreich umgesetzten Vorschläge Kades, die königliche Bibliothek durch Deposita zur Zentralstelle der sächsischen Musikgeschichte zu machen, weist indes eine klare Sammlungs-Motivation auf. Siehe dazu Landmann und Reich, Führer durch die Musikabteilung 1980 (wie Anm. 3), S. 42-44.

12 Zur Geschichte der Berliner Musiksammlung siehe u.a. Rudolf Elvers, „75 Jahre Musikabteilung“, in: Mitteilungen der Staatsbibliothek Preußischer Kulturbesitz 19/20 (1987/88), S. 199-204 und 137-143; Hans-Joachim Schulze, „Karl Friedrich Zelter und der Nachlaß des Bach-Biographen Johann Nikolaus Forkel. Anmerkungen zur Bach-Überlieferung in Berlin und zur Frühgeschichte der Musiksammlung an der königlichen Bibliothek“, in: Jahrbuch des Staatlichen Instituts für Musikforschung Preußischer Kulturbesitz (1993), S. 141-150; und - in einem größeren Kontext - Martina Rebmann, „Die Musikabteilung der Staatsbibliothek zu Berlin - Preußischer Kulturbesitz. Ein Kompetenz-Zentrum für Musik in nationalbibliothekarischem Zusammenhang“, in: Fontes artis musicae 58 (2011), S. 244-252.

13 Siehe etwa Wilhelm Virneisel, „Otto Nicolai als Musiksammler“, in: Festschrift Max Schneider zum 80. Geburtstag, hrsg. von Walther Vetter, Leipzig 1955, S. 227-240; „Aloys Fuchs als Sammler Bachscher Werke“, in: Bach-Jahrbuch 47 (1960), S. 83-99; Richard Schaal, „Neues zur Biographie von Friedrich August Grasnick. Ein Beitrag zur Musiküberlieferung der Berliner Staatsbibliothek“, in: Im Dienst der Quellen zur Musik. Festschrift Gertraut Haberkamp zum 65. Geburtstag, hrsg. von der Bischöflichen Zentralbibliothek Regensburg durch Paul Mai, Tutzing 2002, S. 521-526; Catalog der Sammlung alter Musik des k. k. Hofrathes Raphael Georg Kiesewetter, Edlen von Wiesenbrunn in Wien, Wien 1847. 
unterscheiden: Stehen im letzteren Fall die Quellen in einem historisch-generischen Verhältnis zueinander und weisen oft auch Verknüpfungen mit weiteren Sammlungsbeständen der Bibliothek und vor allem des lokalen Archivs auf, wird der Bezug bei privaten Sammlungen erst durch die Auswahlentscheidungen des Sammlers hergestellt, der je nachdem besonders auf einen Komponisten, eine Gattung, bestimmte Epochen oder - wie Poelchau - auf einen repräsentativen Querschnitt der Musikgeschichte abhebt.

Poelchau, der wohl bereits in den 1790ern mit dem Sammeln begann, ${ }^{14}$ war bereits kein reiner Liebhaber-Sammler mit bloßem Interesse am kuriosen oder auratischen Einzel-Objekt mehr (obwohl er besonders viel in autographe Komponistenmanuskripte investierte), sondern richtete seine Tätigkeit an einem musikhistorischen Anspruch und einem wahrgenommenen Desiderat aus. Ausgehend von der Diagnose fehlender öffentlicher und privater Sammlungen von „Denkmalen der Tonkunst“ ging es ihm um die Zusammenstellung eines „dem Forscher wie dem angehenden Kunstjünger“ dienenden „Archivs der musikalischen Kunst“ zur Sammlung und Rettung „musikalische[r] Alterthümer", das er durchaus institutionalisiert sehen wollte, wie er in einer Denkschrift von 1823 ausführte. ${ }^{15}$ Zweck dieses Archivs - dessen wissenschaftliche Anlage er mehrmals betont - sollte einerseits die Erforschung der einzelnen Werke und das Studium der Musik in ihrer historischen Entwicklung sein, andererseits Editionen zum Zwecke der Wiederbelebung im Konzertbetrieb. Dasselbe Ziel verfolgten rund 100 Jahre später auch Heinrich Schenker und Anthony van Hoboken mit ihrem „Archiv für Photogramme musikalischer Meister-Handschriften ", ${ }^{16}$ nur, dass es sich hierbei nicht mehr um eine physische Musik-Sammlung handelte, sondern eine - heute würde man sagen - virtuelle.

In jedem Fall arbeiteten sowohl das ,bloße Aufbewahren bzw. das Erhalten von Musikalien wie ihr explizites Sammeln der entstehenden Musikwissenschaft als einer primär historisch ausgerichteten, quellenbasierten und am musikalischen Kunstwerk interessierten Disziplin in grundlegender Weise zu, lieferten sie ihr doch das Material, mit dem die Erforschung der „Kunstgeschichte der Musik“ (Robert Eitner) ${ }^{17}$ überhaupt erst sinnvoll betrieben werden konnte. Andere Zweige der Musikwissenschaft, vor allem die systematische und vergleichende, bedurften für ihre Forschungen anderer ,Arbeitsinstrumente und Materialien und nutzten vorrangig den Bücherbestand der Bibliotheken.

14 In dem in Anmerkung 15 zitierten Text von 1823 spricht er an einer Stelle davon, dass ihn die dort geäußerten Gedanken über den Wert alter Musikalien „schon vor dreißig Jahren“ bestimmt hätten, ein entsprechendes Noten-Archiv anzulegen.

15 Georg Poelchau, Archiv der musikalischen Kunst zur Beförderung eines gründlichen Studiums derselben (1823), in D-B: Acta betr. den Ankauf der musikal. Bibliothek des Musikdirektors Naue zu Halle und der Bibliothek des Musiklehrers Pölchau hierselbst 1823-51, Vol. No. III; H. 1, Bl. 98-106.

16 Text des Gründungsaufrufs von 1927 siehe http://www.schenkerdocumentsonline.org/documents/other/OJ-910_2.html.

17 Robert Eitner, „Ein Sammelband alter Musikalien aus der Stadtbibliothek in Danzig“, in: MfM 2 (1870), S. 81. 


\section{Erschließen}

Auf das Bewahren und Sammeln muss notwendigerweise die Erschließung der Bestände folgen, soll eine Sammlung auch einem wissenschaftlichen Zweck dienen können. Das Gründungsdokument der Dresdner Musikabteilung ist ja denn auch nicht zufällig eine eigene Signaturengruppe Musik samt eigenem Katalog, der dann mit der nicht nur quantitativen, sondern auch qualitativen Erweiterung der Sammlung mehrmals modifiziert oder ganz neu erstellt wurde. Wissenschaftlichen Standards genügende (bzw. diese selbst erst entwickelnde), einen Bestand erschließende und aufbereitende Kataloge sind folglich als die ersten genuinen (musik-)wissenschaftlichen Forschungsergebnisse in Bezug auf Musiksammlungen anzusehen und unterscheiden sich kategorial und funktional von früheren Formen der Musikalien-Verzeichnung wie etwa Katalogen fürstlicher Privatsammlungen oder Nachlassverzeichnissen (s. Tabelle 2).

Zwei Phasen lassen sich dabei unterscheiden: Beginnend mit Johann Nikolaus Forkels Allgemeiner Litteratur der Musik ${ }^{18}$ (der seinerseits an frühere Arbeiten wie etwa Johann Jakob Adlungs Anleitung zur musikalischen Gelahrtheit von 1758 oder die Werklisten in den Musikgeschichten von John Hawkins und Charles Burney anknüpfen konnte) stand in der ersten Hälfte des 19. Jahrhunderts zunächst die musikbezogene Literatur im Fokus. Verschiedene Autoren versuchten einen bibliographischen Überblick über das existierende Musikschrifttum zu geben, es zusammenzustellen und thematisch zu ordnen. Auf das hier Zusammengestellte - neben Forkels Werk fand jedenfalls im deutschsprachigen Raum vor allem die sich eng an Forkel anlehnende Systematisch-chronologische Darstellung der musikalischen Literatur des Leipziger Organisten Carl Ferdinand Becker Beachtung ${ }^{19}$ - bezogen sich dann (Musik-)Bibliothekare bei der Erfassung ihrer jeweiligen Sammlungsbestände. Eberts Dresdner Katalog beispielsweise griff verschiedene Kategorien und Formulierungen aus Forkels und Beckers Systematisierungen auf, passte sie aber teils sehr frei der Struktur seiner eigenen Sammlung an: So fasste er Kapitel zusammen, brachte Großabschnitte in eine andere Reihenfolge (stehen bei Forkel und Becker Werke über den Ursprung, Zweck und die Wirkung von Musik jeweils am Anfang, machen sie bei Ebert erst die dritte Klasse aus) oder griff nur einen jeweils für ihn relevanten Unterbereich heraus. Insgesamt ergibt sich damit aber eine durchaus gut nachvollziehbare Systematik mit neun sogenannten Klassen (s. Tabelle 3).

Musikalische Werke kommen bei Ebert nur als chronologisch geordnete neunte Klasse am Ende seines Kataloges vor (und umfassen insgesamt nur 30 Folio-, neun Quarto- und vier Octavo-Nummern). In der zweiten Hälfte des 19. Jahrhunderts gerieten dann jedoch zunehmend die Musikalien selbst und ihre Ordnung und Verzeichnung in den bibliothekarischen Fokus. Schon Forkel hatte ein auf ,musica practica' bezogenes Pendant seiner Literatursammlung als

18 Johann Nikolaus Forkel, Allgemeine Litteratur der Musik oder Anleitung zur Kenntniß musikalischer Bücher, welche von den ältesten bis auf die neusten Zeiten bey den Griechen, Römern und den meisten neuern europäischen Nationen sind geschrieben worden, Leipzig 1792, Reprint Hildesheim ${ }^{2} 2001$. Siehe dazu Oliver Wiener, „Bibliothek und Probe im Umbruch des disziplinären Feldes. Voraussetzungen musikwissenschaftlichen Sammelns und Verzeichnens im 18. Jahrhundert“, in: Inventar und Werkverzeichnis. Ordnung und Zählung als Faktoren der Rezeptionsgeschichte, hrsg. von Thomas Hochradner und Dominik Reinhardt, Freiburg i. Br. 2011, S. 111-126 (klang-reden. Schriften zur Musikalischen Rezeptions- und Interpretationsgeschichte. 7).

19 Carl Ferdinand Becker, Systematisch-chronologische Darstellung der musikalischen Literatur von der frühesten bis auf die neueste Zeit, Leipzig 1836. 
Desiderat benannt und gleich einen Vorschlag zu einer Klassifikation gemacht, der alphabetische mit gattungsbezogenen und chronologischen Aspekten verschränkte (Hauptklassen alphabetisch: 1. Kirchen-Komponisten, 2. Theater-Komponisten, 3. Kammer-Komponisten, 4. Virtuosen, 5. Instrumentenbauer, Unterklassen nach Gattungen, innerhalb derer dann chronologisch angeordnet werden solle). ${ }^{20}$

Meist waren die Kataloge zunächst handschriftlich (also nur vor Ort einsehbar), wurden dann teilweise später gedruckt (also verbreitet) und / oder in größere bibliographische oder quellenbezogene Publikationen eingearbeitet. Für die Zeit um 1900 ist hier natürlich vor allem das monumentale Biographisch-bibliographische Quellenlexikon der Musiker und Musikgelehrten von Robert Eitner zu nennen. Daneben stehen die Monatshefte für Musikgeschichte (MfM, bereits 1869 von Eitner initiiert), die als einem von vier Zielen auch der bibliographischen Erschließung von Musik gewidmet sein sollten. ${ }^{21}$ Mit solchen Medien bildeten und etablierten sich zugleich übergreifende Standards der Quellen-Erschließung und -Kategorisierung. Denn wie Musik sinnvoll zu ordnen und zu kategorisieren sein könnte, welche Aspekte der Quellen (und der erfassten Musikstücke) wie Erwähnung finden und ob dabei jeweils vielleicht implizit schon Forschungsinteressen Berücksichtigung finden, sind Fragen, die sich mit den ersten bibliothekarischen Musik-Katalogen überhaupt erst stellten und durchaus verschiedene Antworten fanden.

Als Medium für solche Aushandlungsprozesse lassen sich beispielsweise Rezensionen zu Katalog-Veröffentlichungen in den MfM lesen: Eitner machte diese Funktion in seiner Besprechung des von Joseph Müller veröffentlichten Katalogs der Königsberger Musikalien-Sammlung im zweiten Jahrgang seiner Zeitschrift sogar explizit: „[Da] die Einrichtung eines brauchbaren Kataloges aber eine unserer Kardinalfragen betrifft, so ist es gewiss von Interesse eine öffentliche Besprechung über diesen Gegenstand herbeizuführen, um aus den verschiedenen Ansichten zur möglichst vollkommensten Einrichtung zu gelangen. “22 Er schließt seinen Text mit „einige[n] Worte[n] über die Anlage eines Kataloges einer grösseren musikalischen Bibliothek“ und der „Anregung zu weiterem Meinungsaustausche“. ${ }^{23}$ Der damalige Hauptdissens scheint - neben einigen terminologischen Fragen wie etwa der Reichweite des Begriffs „Sammelwerk“ - in der Frage der Anordnung bestanden zu haben: Denn während Müller seine Sammelwerke nach Gattungen bzw. Funktionen zu trennen unternimmt („Tonwerke für die Kirche“, also geistliche Musik, neben „Tonwerken für das Haus“, also weltliche bzw. Kammer-Musik), verweist Eitner auf das grundsätzliche Problem übergreifender Kategorien für Musik und die häufige Zusammenbindung mehrerer Gattungen in einer Quelle und spricht sich daher dezidiert für eine alphabetische Anlage (vor sachlicher oder chronologischer) aus: „Nach meiner Ansicht muss ein Katalog gar keinen Index bedürfen, da er ja selbst ein Index sein soll; um dies aber zu erreichen, muss er auch wie ein Register angelegt sein. ${ }^{\prime 24}$

20 Forkel, Allgemeine Litteratur 1792 (wie Anm. 18), Vorrede S. VII f.

21 Siehe „Programm“ in Nr. 1, 1869, S. 1: „4) Bibliographische Arbeiten: a. Cataloge von öffentlichen und privaten Bibliotheken. b. Verzeichnisse von neueren und neuen Werken über Musik im Anschlusse an Forkel u. Becker. c. Kritiken über alle neuen musikliterarischen Erzeugnisse, sowie über alle Erscheinungen, welche die Musikgeschichtsforschung betreffen“.

22 Robert Eitner, „Die Königsberger Musikbibliothek“, in: MfM 2 (1870), S. 146-148, hier S. 146.

23 Ebd., S. 148.

24 Ebd., S. 147. 
Er kommt dann auch noch auf die Erschließungstiefe eines Katalogs zu sprechen und empfiehlt hier Müllers damals offenkundig ungewöhnliche Entscheidung, neuere Werke genauso ausführlich zu erfassen wie ältere, als Norm. Die als Frucht historischen Interesses aufgekommene bevorzugende Beschäftigung mit den älteren Quellen tut er als antiquarische Liebhaberei ab und gibt zu bedenken, „dass das jetzt Neue in hundert Jahren zum Alten gehört, und unsere Nachkommen durch solche antiquarische Liebhabereien in gleicher Weise beeinträchtigt wären, als es von unseren Vorfahren uns gegenüber geschehen ist. ${ }^{\text {"25 }}$ Die Trennung in alte und neue Musik findet sich in musikhistorischen Systematisierungen der Zeit immer wieder - der Beginn der modernen Musik wird dabei meist um 1700 angesetzt - und ist meist konstitutiv für Sammlungsstrategien oder die Anlage von Katalogen und zeugt zugleich von den Paradigmen, unter denen sich ein musikhistorisches Bewusstsein entwickelte.

Am Ende dieser Besprechung schlägt Eitner Folgendes als Standard für Kataloge größerer Musiksammlungen vor: Haupt-Unterteilung in drei große Kategorien, nämlich „literarische Werke“ (also musikbezogene Literatur), „praktische Musik“ (also Musikalien) und „Hymnologie“ (Gesangbücher). Diese seien dann jeweils alphabetisch zu ordnen, bei nicht eindeutigen Fällen mit Mehrfachverzeichnung. Zur weiteren Verbesserung der Auffindbarkeit könne ein Sachregister mit Jahresangaben ergänzt werden. ${ }^{26}$ In seinen eigenen Katalogisierungsarbeiten - allen voran dem gemeinsam mit Otto Kade erarbeiteten Katalog der Dresdner Musikalien - setzte er diese Auffassungen jeweils um. ${ }^{27}$

Verwandt mit der Diskussion um die Ordnung von Katalogen sind die Bemühungen um orthographische Standardisierungen von Namen: ${ }^{28}$ Die musikwissenschaftlich und musikbibliographisch Tätigen und ihre verschiedenen Erschließungsprojekte hatten so sehr an Zahl zugenommen, dass uneinheitliche Schreibweisen und allzu disparate Ordnungsstrategien offenbar den allgemeinen Nutzen der jeweiligen Vorhaben gefährdeten.

Nicht zuletzt durch Eitners Aktivitäten setzte sich die Überzeugung davon durch, dass eine umfängliche Erschließung und Inventarisierung von Quellen die Voraussetzung jeglicher musikhistorischen Forschung sein müsse. Die für die Ordnung herangezogenen Kriterien weisen jeweils auf zugrunde liegende, teils konkurrierende historiographische Konzepte, in erster Linie die beiden Haupt-Optionen, entweder nach Personen (Komponisten-zentrierte Musikgeschichtsschreibung) oder nach Gattungen (funktions- und kontextbezogene Musikgeschichtsschreibung) zu ordnen. Quellen- bzw. Material-basierte Kriterien wie Format und Quellentypus (Manuskript versus Druck) interagierten damit auf je spezifische Weise.

25 Ebd., S. 148.

26 Ebd.

27 Robert Eitner und Otto Kade, Katalog der Musik-Sammlung der Kgl. öffentlichen Bibliothek zu Dresden, Leipzig 1890 (Beihefte zu den Monatsheften für Musikgeschichte. 22).

28 Siehe dazu etwa die entsprechende Mitteilung in MfM 20 (1888), S. 73. 


\section{Erforschen}

Die Existenz und die Form von Katalogen hatten einen unmittelbaren Einfluss auf den Beginn und die Art der forscherischen Beschäftigung mit den jeweiligen Sammlungsbeständen. Während handschriftliche Kataloge oft nur ortsansässige Personen erreichten und insofern vor allem für Regionalforschung ausgewertet wurden, ermöglichten es gedruckte Kataloge hingegen auch auswärtigen Wissenschaftlern, sich mit den Beständen zu beschäftigen: sei es, dass sie zu den entsprechenden Orten reisten, oder dass sie Titel (oder Kopien von ihnen) ausliehen.

Gerade zu Beginn der hier beschriebenen Entwicklungen traten freilich Sammler, Kustoden und Bibliothekare auch als die ersten Erforscher ihrer Bestände in Erscheinung: Fürstenau, Kade oder Eitner publizierten nicht nur bibliographische Arbeiten, sondern auch im engeren Sinne musikhistorische.

An einer Person wie Siegfried Dehn in Berlin indes zeigt sich bereits die bald eintretende und als Zeichen zunehmender Professionalisierung und Formalisierung zu wertende Rollentrennung zwischen eher bibliographisch arbeitenden Bibliothekaren und historiographisch arbeitenden Musikwissenschaftlern. Dehn veröffentlichte nämlich im Grunde ausschließlich Noteneditionen, u.a. die Bände 15-23 der bei Peters erscheinenden Bach-Ausgabe, für die er wesentlich auf die Bestände der Berliner Bibliothek zurückgreifen konnte. Sein Nachfolger Franz Espagne machte es ebenso und engagierte sich besonders in den Beethoven- und Palestrina-Editionen.

Kritische, quellengestützte Editionen sind - wie erwähnt - schon von Poelchau als gleichsam ,natürliche' Auswertungsform von musikalischen Quellensammlungen benannt und in Angriff genommen worden. Eitner teilte diesen Gedanken und flankierte die Bestandsübersichten in den Monatsheften für Musikgeschichte mit den editorisch ausgerichteten Publikationen der Gesellschaft für Musikforschung.

Hinzu kamen die großen, in den 1890er Jahren begründeten und meist national motivierten Denkmäler-Reihen, deren Durchführung vor der Ordnung und Erschließung der großen regionalen Musik-Sammlungen nicht nur nicht möglich, sondern nicht einmal denkbar gewesen wäre: Im deutschsprachigen Gebiet folgte auf die von Preußen initiierten Denkmäler Deutscher Tonkunst (1892) und die Denkmäler der Tonkunst in Österreich (1894) bereits als dritte Umsetzung die Musik am sächsischen Hofe (1898). ${ }^{29}$

Editionen und Faksimile-Ausgaben - und damit der Bereich des Vervielfältigens der eigenen Quellen - wurden im Laufe des 20. Jahrhunderts dann ein reguläres Standbein musikbibliothekarischer Arbeit: Dresden beispielsweise war seit den 1970er Jahren Partner der Reihe Peters Reprints (für Theoretica und Musikwerke) und Initiator der Faksimile-Reihe Musik der Dresdner Hofkapelle. Als aktuelle Erscheinungsform dieser Vervielfältigungs-Bestrebungen wären die modernen Digitalisierungs-Projekte zu nennen.

Gerade vor Ort gewachsene und eine Musiktradition dokumentierende Sammlungen haben von Anfang an primär lokalgeschichtliche Forschung inspiriert. Die konkrete Ausrichtung vieler übernehmender Bibliotheken als Landesbibliothek mit dem Auftrag, die regionale Geschichte zu

29 Andere frühe Projekte: Uitgave van Oudere Noord-Nederlandse Meesterwerken (1869-1939), The Old English Edition (1889-1902), Les maîstres musiciens de la renaissance française (1894-1908), Archives des maîtres de l'orgue (1898-1911), Denkmäler der Tonkunst in Bayern (DDT, II. Folge), ab 1900. 
dokumentieren, hat diese Tendenz zugleich erleichtert und noch einmal verstärkt. Die Identifizierung von politischen mit kulturellen Einheiten, wie es durch die politische Landkarte Deutschlands in Verbindung mit der gerade auch kulturell ausgetragenen Konkurrenz der Fürsten und Höfe nahegelegt wurde, stellte die Basis dafür dar. Somit entsprechen Pläne wie derjenige Otto Kades, die Dresdner Musiksammlung durch Deposita und Ankäufe zum Dokumentationszentrum bzw. zur „Centralstelle“ der sächsischen Musikgeschichte mit besonderer Berücksichtigung der Musikpraxis am Dresdner Hof zu machen, vollauf dem Zeitgeist. Bibliotheks- und Archivfunktion verschränken sich hier miteinander.

Musiksammlungen sind gegenüber musikwissenschaftlichen Instituten und Studiengängen die ältere musikwissenschaftliche Institution. Entsprechend besteht die erste Generation von Forschern aus Personen ohne formalisierte (musik)wissenschaftliche oder historische Ausbildung (darauf wird gleich noch einmal zurückzukommen sein): So war Moritz Fürstenau bekanntlich Flötist in der sächsischen Hofkapelle, erhielt dann zusätzlich den Posten als Kustos der königlichen Privat-Musikaliensammlung und wertete die ihm anvertrauten Bestände u. a. für seine zweibändige Geschichte der Musik und des Theaters am Hofe zu Dresden aus. Auch Julius Rühlmann und Wilhelm Joseph von Wasielewski, die in den 1860er und 70er Jahren mit den Beständen arbeiteten, waren Hofmusiker. Dasselbe gilt für Otto Kade in Schwerin. Paul Hermann Otto von Waldersee, der als einer der ersten Darmstädter Musikalienbestände untersuchte, war ursprünglich Militärmusiker. Auch Privatgelehrte verschiedener Hintergründe waren darunter, so etwa Emil Roth in Darmstadt.

Mit der Etablierung einer regulären Musikwissenschaft in Sachsen unter Hugo Riemann (Berufung nach Leipzig 1901, Gründung des Instituts 1908) professionalisierte sich auch die Erforschung der Dresdner Musikalien-Bestände. Im frühen 20. Jahrhundert qualifizierten sich reihenweise junge Musikwissenschaftler (und einige wenige Musikwissenschaftlerinnen) mit Dissertationen, die systematisch das Dresdner Repertoire erforschten (und dabei im Einklang mit den herausgearbeiteten historiographischen Paradigmen zumeist eine erste Unterteilung in Komponisten und dann in Werkgruppen vornahmen) (s. Tabelle 4).

Dieselbe Entwicklung ist auch andernorts zu beobachten: An der Darmstädter Bibliothek wirkte ab 1919 mit Friedrich Noack ein promovierter Musikwissenschaftler als Musikbibliothekar (seine Dissertation hatte die Darmstädter Graupner-Bestände ausgewertet). Und Wilibald Nagel, der die Bestände ebenfalls intensiv nutzte, wurde nach einer musikhistorischen Habilitation Professor für Musikwissenschaft an der Stuttgarter Musikhochschule.

Das stetig zunehmende Forschungsvolumen, die Etablierung von Schriftenreihen, Zeitschriften und Editionsreihen zog dann wiederum eine Bestandserweiterung der Musiksammlungen und eine gewisse De-Regionalisierung nach sich: Aus vornehmlich historisch-dokumentarischen Sammlungen wurden so recht schnell die wissenschaftlichen Arbeitsinstrumente mit der typischen Bestandsmischung aus Original-Quellen, Faksimilia, Editionen und Sekundärliteratur, wie wir sie auch heute noch kennen. 


\section{Personalien}

Den Abschluss mögen einige weitere Bemerkungen zum Personal der beschriebenen Entwicklungen bilden. Die Viten der ersten Musik-Sammler, -Kustoden, -Bibliothekare und -Wissenschaftler sind nämlich im Hinblick auf die Institutionalisierung des Faches ausgesprochen instruktiv. Als Hilfskonstruktion sei eine Unterscheidung in drei (nicht in jedem Fall chronologisch voneinander getrennte) Generationen vorgeschlagen:

Die erste Generation wird ganz überwiegend aus professionellen Musikern und Personen mit musikalischer Ausbildung gebildet, hinzu kommen Privatiers, Staatsbeamte und andere Personen mit nicht-musikalischem Hintergrund, die dann auf verschiedensten Wegen ein musikantiquarisches und musikhistorisches Interesse ausbilden, es aber privat und ohne institutionelle Einbindungsmöglichkeit verfolgen. In dieser Generation finden wir vor allem Musiksammler wie Poelchau, Proske, ${ }^{30}$ Kiesewetter $^{31}$ oder Fuchs ${ }^{32}$ sowie frühe Musikhistoriker und -biographen. Zwischen ihnen bestanden durchaus dichte Netzwerke, die aber - strukturell gesehen - im Wesentlichen einen privaten Charakter aufwiesen. ${ }^{33}$

Die zweite Generation verfügte dann ebenfalls noch nicht über eine formale musikwissenschaftliche Ausbildung, konnte aber ihr Interesse zum Hauptberuf oder doch wenigstens Nebenamt machen: Hier wären Fürstenau, Eitner und Kade ebenso zu nennen wie Siegfried Dehn, Alfred Wotquenne oder Friedrich Noack sowie die erste Generation von Musikwissenschafts-Professoren. Sowohl durch ihre Einbindung in meist staatliche Institutionen als auch verschiedene vereinheitlichende und vernetzende Maßnahmen schufen sie überhaupt erst ein Interaktionsfeld Musikforschung. Dieses dokumentierte sich strukturell in der Gründung von Fachgesellschaften, Periodika und weiteren Gemeinschaftsvorhaben wie Editions- und Schriftenreihen und führte zur Etablierung übergreifender wissenschaftlicher Standards, zur Herausbildung bzw. Adaption fachspezifischer Methoden, zu von vielen verfolgten thematischen Interessen, Schwerpunkten und Erschließungsweisen.

Diese Personengruppe wurde dann zu den Lehrern der dritten Generation, die erstmals in den Genuss eines grundständigen musikwissenschaftlichen Studiums kam, in dem sich die gerade genannten übergreifenden Standards materialisiert hatten. Zugleich konnte diese Generation auf die Fundamentierungsarbeit der vorangegangenen Generationen zurückgreifen, also auf Sammlung, Kataloge, Editionen, und diese in historiographischen Arbeiten auswerten und fruchtbar werden lassen.

$30 \mathrm{Zu}$ Proskes Biographie und seiner Sammlung siehe die Beiträge in: Musica divina. Ausstellung zum 400. Todestag von Giovanni Pierluigi da Palestrina und Orlando di Lasso und zum 200. Geburtstag von Carl Proske, Regensburg 1994.

31 Zu Kiesewetter siehe Herfrid Kier, Raphael Georg Kiesewetter (1773-1850). Wegbereiter des musikalischen Historismus, Regensburg 1968 (Studien zur Musikgeschichte des 19. Jahrhunderts. 13).

32 Zu Fuchs und seiner Sammeltätigkeit siehe Richard Schaal, Quellen und Forschungen zur Wiener Musiksammlung von Aloys Fuchs, Wien 1966; sowie Richard Schaal, Die Tonkünstler-Porträts der Wiener Musiksammlung von Aloys Fuchs, Wilhelmshaven 1970.

33 Zur Beziehung zwischen Fuchs und Poelchau siehe etwa Till Reininghaus, „Zwischen Wien und Berlin. Die Musiksammlungen von Aloys Fuchs und Georg Poelchau im Spiegel ihrer Korrespondenz“, in: Wasserzeichen Schreiber - Provenienzen. Neue Methoden zur Erforschung und Erschließung von Kulturgut im digitalen Zeitalter, hrsg. von Wolfgang Eckhardt u.a., Frankfurt a. M. 2016 (Zeitschrift für Bibliothekswesen und Bibliographie, Sonderband. 118), S. 27-45. 
Dieser dreischichtige Prozess der Standardisierung, Professionalisierung und Institutionalisierung wirkte zudem wiederum auf die Betreuung der Musik-Sammlungen zurück: Waren zunächst, wenn überhaupt, entweder Musiker oder allgemeine Bibliothekare zu Kustoden bestellt worden, standen nun spezialisierte und wissenschaftlich qualifizierte Musikbibliothekare zur Verfügung und wurden auch zunehmend eingestellt. Die Ausdifferenzierung von bibliographisch-antiquarischen Berufsbildern einerseits und musikhistorischen und -wissenschaftlichen andererseits ist ebenfalls eine Folge der Institutionalisierung der Musikwissenschaft - und führt(e) im besten Falle zu einem ausgesprochen fruchtbaren Miteinander. ${ }^{34}$

Musiksammlungen als von zunächst individuellem, dann immer allgemeiner werdendem musikhistorischen Bewusstsein geschaffene Fakten spielten also eine maßgebliche, noch längst nicht hinlänglich erforschte Rolle in der Geschichte der Musikwissenschaft und dürfen - gemeinsam mit einigen wenigen anderen Entwicklungen - geradezu als ihre Katalysatoren gelten.

34 An dieser Stelle möchte ich den Leiterinnen der Berliner und Dresdner Musikabteilungen, Frau Dr. Martina Rebmann und Frau Dr. Barbara Wiermann, sehr herzlich für ihre freundliche Ansprechbarkeit und ihre Hilfsbereitschaft während der Arbeit an diesem Text danken. 
Tab. 1: Private Musiksammlungen und ihr Aufgehen in öffentlichen Sammlungen (Auswahl)

\begin{tabular}{|c|c|c|c|}
\hline Sammler & Übernehmender Sammler & $\begin{array}{l}\text { Übernehmende } \\
\text { Sammlung }\end{array}$ & Ausrichtung \\
\hline $\begin{array}{l}\text { Carl Philipp Emanuel Bach } \\
(1714-1788)\end{array}$ & $\begin{array}{l}\text { Georg Poelchau } \\
(1773-1836)\end{array}$ & $\begin{array}{l}\text { 1841: Königliche } \\
\text { Bibliothek Berlin }\end{array}$ & $\begin{array}{l}\text { Altbachisches Archiv, } \\
\text { Porträts, Autographen } \\
\text { und Abschriften, Drucke } \\
\text { und Musiktheorie }\end{array}$ \\
\hline $\begin{array}{l}\text { Raphael Georg Kiesewetter } \\
(1773-1850)\end{array}$ & & $\begin{array}{l}\text { Österreichische } \\
\text { Nationalbibliothek }\end{array}$ & v. a. alte Musik \\
\hline $\begin{array}{l}\text { Friedrich August Gotthold } \\
(1778-1858)\end{array}$ & & $\begin{array}{l}\text { 1858: Universitätsbiblio- } \\
\text { thek Königsberg }\end{array}$ & \\
\hline $\begin{array}{l}\text { Fortunato Santini } \\
(1778-1861)\end{array}$ & & $\begin{array}{l}\text { 1862: Münster, West- } \\
\text { fälische Akademie- und } \\
\text { Landesbibliothek }\end{array}$ & Kirchenmusik \\
\hline Carl Proske (1794-1861) & & $\begin{array}{l}\text { 1861: Bischöfliche Zen- } \\
\text { tralbibliothek Regensburg }\end{array}$ & $\begin{array}{l}\text { Abschriften und Origi- } \\
\text { nale alter italienischer } \\
\text { Kirchenmusik }\end{array}$ \\
\hline Aloys Fuchs (1799-1853) & $\begin{array}{l}\text { Friedrich August } \\
\text { Grasnick (1798-1877) }\end{array}$ & $\begin{array}{l}\text { 1879: Königliche } \\
\text { Bibliothek Berlin }\end{array}$ & Autographen, Porträts \\
\hline $\begin{array}{l}\text { Siegfried Dehn } \\
(1799-1858)\end{array}$ & & $\begin{array}{l}\text { 1959: Staatsbibliothek } \\
\text { Berlin }\end{array}$ & \\
\hline $\begin{array}{l}\text { Victor Schoelcher } \\
(1804-1893)\end{array}$ & $\begin{array}{l}\text { Friedrich Chrysander } \\
(1826-1901)\end{array}$ & $\begin{array}{l}\text { 1868/75: Stadtbibliothek } \\
\text { Hamburg }\end{array}$ & Händel-Sammlung \\
\hline Otto Nicolai (1810-1849) & & $\begin{array}{l}\text { Königliche Bibliothek } \\
\text { Berlin }\end{array}$ & \\
\hline $\begin{array}{l}\text { Guido Richard Wagner } \\
(1822-1896)\end{array}$ & & $\begin{array}{l}\text { 1902: Königliches } \\
\text { Konservatorium Brüssel } \\
\text { (Wotquenne) }\end{array}$ & \\
\hline $\begin{array}{l}\text { Alfred Wotquenne } \\
(1867-1939)\end{array}$ & $\begin{array}{l}\text { 1913: Versteigerung eines } \\
\text { Teils }\end{array}$ & $\begin{array}{l}\text { 1929: Verkauf der wert- } \\
\text { vollsten Stücke an die } \\
\text { Library of Congress }\end{array}$ & \\
\hline $\begin{array}{l}\text { Anthony van Hoboken } \\
(1887-1983)\end{array}$ & & $\begin{array}{l}\text { 1974: Österreichische } \\
\text { Nationalbibliothek }\end{array}$ & $\begin{array}{l}\text { Erst- und Frühdrucke, } \\
\text { Musiktheorie von Bach } \\
\text { bis Brahms }\end{array}$ \\
\hline $\begin{array}{l}\text { Max Hellmuth } \\
(1904-1945)\end{array}$ & & Landesbibliothek Coburg & \\
\hline
\end{tabular}


Tabelle 2: Frühe Kataloge von Musiksammlungen (Auswahl)

\begin{tabular}{|c|c|c|c|c|}
\hline Ort & Jahr & Bearbeiter & Hs. Katalog & Gedr. Katalog \\
\hline \multirow[t]{4}{*}{ Dresden } & 1816 & Friedrich Ebert & $\mathrm{x}$ & \\
\hline & $1868 \mathrm{f}$. & Moritz Fürstenau & $\mathrm{x}$ & \\
\hline & 1890 & Robert Eitner, Otto Kade & & $\mathrm{x}$ \\
\hline & $1926-30$ & & $\mathrm{x}$ & \\
\hline Wien & 1847 & Raphael Georg Kiesewetter & & $\mathrm{x}$ \\
\hline Hamburg & $1855-57$ & Carl Rudolf Wilhelm Klose & $\mathrm{x}$ & \\
\hline Einsiedeln & $1860 \mathrm{er}$ & unbekannt & $\mathrm{x}$ & \\
\hline Leipzig & 1868 & Alfred Dörffel & & $\mathrm{x}$ \\
\hline Danzig & vor 1872 & unbekannt & $\mathrm{x}$ & \\
\hline Königsberg & 1870 & Joseph Müller & & $\mathrm{x}$ \\
\hline \multirow[t]{2}{*}{ Darmstadt } & 1874 & Philipp Alexander Walther & & $\mathrm{x}$ \\
\hline & $1920-27$ & Friedrich Noack & $\mathrm{x}$ & \\
\hline München & 1879 & Julius Joseph Maier & & $\mathrm{x}$ \\
\hline Augsburg & 1879 & Hans Michel Schletterer & $\mathrm{x}$ & $\mathrm{x}(\text { in } M f M)^{\star}$ \\
\hline Göttingen & $1879 / 1883$ & Albert Quantz & & $\mathrm{x}($ in $M f M)$ \\
\hline Lübeck & ab 1883 & Carl Stiehl & $\mathrm{x}$ & \\
\hline Breslau & $1883 / 1890$ & Emil Bohn & $\mathrm{x}$ & \\
\hline Wolfenbüttel & 1890 & Emil Vogel & & $\mathrm{x}($ in $M f M)$ \\
\hline Basel & $1891 \mathrm{f}$. & E. Julius Richter & & $\mathrm{x}($ in $M f M)$ \\
\hline Schwerin & $1893 / 1899$ & Otto Kade & & $\mathrm{x}$ \\
\hline Sorau & 1901 & & & $\mathrm{x}($ in $M f M)$ \\
\hline Stuttgart & $1901 \mathrm{f}$. & August Halm & & $\mathrm{x}($ in $M f M)$ \\
\hline
\end{tabular}

${ }^{\star} M f M=$ Monatshefte für Musikgeschichte 
Tabelle 3: Klassifikation der Musik-Literatur in Eberts Katalog der Dresdner Musiksammlung und Vergleich mit Forkels Systematisierung in der Allgemeinen Litteratur der Musik

\begin{tabular}{|c|c|c|}
\hline \multicolumn{2}{|l|}{ Ebert } & \multirow[t]{2}{*}{ Forkel } \\
\hline Klassen & Unterabteilungen, Anmerkungen & \\
\hline $\begin{array}{l}\text { I. Geschichte und Bücherkunde } \\
\text { der Musik }\end{array}$ & $\begin{array}{l}\text { a. allgemeine und Vergleichung der alten und neuen } \\
\text { Musik } \\
\text { b. Geschichte der alten Musik } \\
\text { c. Geschichte der neuen Musik } \\
\text { d. Lebensbeschreibungen von Musikern }\end{array}$ & $\mathrm{I} / 2-7$ \\
\hline \multicolumn{3}{|l|}{$\begin{array}{l}\text { II. Vermischte Schriften, Samm- } \\
\text { lungen, Journale }\end{array}$} \\
\hline $\begin{array}{l}\text { III. Einleitungsschriften über } \\
\text { Natur, Geist, Einfluß, Nutzen } \\
\text { p.p. der Musik }\end{array}$ & & $\mathrm{I} / 1$ \\
\hline \multicolumn{3}{|l|}{$\begin{array}{l}\text { IV. Wörterbücher, Systeme und } \\
\text { Compendien der gesammten } \\
\text { Musik }\end{array}$} \\
\hline $\begin{array}{l}\text { V. Einzelne Materien aus dem } \\
\text { Gebiete der gesammten Musik }\end{array}$ & $\begin{array}{l}\text { a. Musikalische Zeichenlehre, Tabulatur } \\
\text { b. Guidonische Solmisation } \\
\text { c. Takt } \\
\text { d. Tonarten } \\
\text { e. Temperatur } \\
\text { f. Harmonie } \\
\text { g. Intervallen } \\
\text { h. Tonleitern } \\
\text { i. Accorde } \\
\text { k. Generalbaß } \\
\text { l. Composition } \\
\text { m. Contrapunct und Fuge } \\
\text { n. Melodie } \\
\text { o. Phantasie } \\
\text { p. musikalischer Vortrag, Ausdruck p.p. }\end{array}$ & $\mathrm{II} / 2,1, \mathrm{II} / 4-5$ \\
\hline $\begin{array}{l}\text { VI. Einzelne Gattungen der } \\
\text { Musik }\end{array}$ & Kirchenmusik nebst Choral, Theatermusik & $\mathrm{I} / 6,2-3$ \\
\hline VII. Singkunst & $\begin{array}{l}\text { a. Aesthetik, Geschichte und Theorie des Gesanges } \\
\text { b. Gesangschulen }\end{array}$ & $\mathrm{II} / 2,2$ \\
\hline $\begin{array}{l}\text { VIII. Schriften über musika- } \\
\text { lische Instrumente }\end{array}$ & $\begin{array}{l}\text { a. über musikalische Instrumente überhaupt } \\
\text { b.-n. einzelne Instrumente }\end{array}$ & $\mathrm{II} / 2,3$ \\
\hline IX. Compositionen & In chronologischer Reihenfolge & \\
\hline
\end{tabular}


Tabelle 4: Frühe Dissertationen zur Dresdner Hofmusik (Auswahl)

\begin{tabular}{|l|l|l|l|l|}
\hline Jahr & Dissertant/in & Thema & $\begin{array}{l}\text { Diss. } \\
\text { Leipzig }\end{array}$ & $\begin{array}{l}\text { Diss. an } \\
\text { anderem } \\
\text { Ort }\end{array}$ \\
\hline 1906 & Carl Mennicke & Hasse und Graun: Sinfonien & ja & \\
\hline 1908 & Bernhard Engelke & Fasch: Leben und Vokalwerke & ja & \\
\hline 1910 & Lucian Kamienski & Hasse: Oratorien & & Berlin \\
\hline 1910 & Georg Felix Kaiser & Weber als Musikschriftsteller & ja & \\
\hline 1911 & Bruno Studeny & Violinsonate im 18. Jahrhundert & & München \\
\hline 1911 & Walther Müller & Hasse: Kirchenmusik & ja & \\
\hline 1913 & Gustav A. Seibel & Heinichen: Leben und Werk & ja & \\
\hline 1916 & Richard Tanner & Heinichen: Opern & ja & \\
\hline 1916 & Richard Engländer & Naumann: Opern & & Berlin \\
\hline 1917 & Charlotte Spitz & Lotti: Opern & & München \\
\hline 1918 & Kurt Kreiser & Reissiger: Leben, Konzertwesen & ja & \\
\hline 1931 & Margarete Högg & Gesangskunst Faustina Bordoni & & Berlin \\
\hline 1932 & Clemens August Schneider & Fasch: Sonaten & & Münster \\
\hline
\end{tabular}

